\title{
Adaptive Moving Sliding Mode Control for SISO Systems: Application to an Electropneumatic System
}

\author{
Assil Ayadi, ${ }^{1}$ Soufien Hajji, ${ }^{1}$ Mohamed Smaoui, ${ }^{2}$ and Abdessattar Chaari ${ }^{1}$ \\ ${ }^{1}$ Laboratory Lab-STA, LR11ES50, National School of Engineering of Sfax, University of Sfax, BP 1173, 3038 Sfax, Tunisia \\ ${ }^{2}$ University of Lyon, Villeurbanne, France \\ Correspondence should be addressed to Assil Ayadi; ayadiassil@yahoo.fr
}

Received 28 November 2015; Revised 31 March 2016; Accepted 6 April 2016

Academic Editor: Manuel Pineda-Sanchez

Copyright (C) 2016 Assil Ayadi et al. This is an open access article distributed under the Creative Commons Attribution License, which permits unrestricted use, distribution, and reproduction in any medium, provided the original work is properly cited.

\begin{abstract}
This paper aims to propose and develop an adaptive moving sliding mode controller (AMSMC) that can be applied for nonlinear single-input single-output (SISO) systems with external disturbances. The main contribution of this framework consists to overcome the chattering phenomenon problem. The discontinuous term of the classic sliding mode control is replaced by an adaptive term. Moreover, a moving sliding surface is proposed to have better tracking and to guarantee robustness to the external disturbances. The parameters of the sliding surface and the adaptive law are deduced based on Lyapunov stability analysis. An experimental application of electropneumatic system is treated to validate the theoretical results.
\end{abstract}

\section{Introduction}

Fluid systems, especially the pneumatic systems, possess a profoundly nonlinear nature at different levels. The complexity of these systems incites researchers to study their behavior and synthesize adequate control laws.

In recent years, many researchers have been interested in robust control applied to electropneumatic actuators. In this context, we can cite, for example, feedback linearization [1], fuzzy control algorithms [2], adaptive control [3], backstepping control [4], and sliding mode control $[5,6]$.

The classic sliding mode operates with high accuracy and robustness with respect to various uncertainties and external disturbances. However, it suffers from a specific drawback: the chattering phenomenon. This phenomenon is caused by the discontinuous term of the control law which is characterized by high oscillations around the sliding manifold. In order to reduce the chattering phenomenon, there are various proposed solutions. In [7-11], high order sliding mode control is proposed. This algorithm ensures that the sliding variable and its consecutive derivatives tend to zero in the presence of the disturbances/uncertainties. The high sliding mode control allows, also, increasing the accuracy of the sliding variable stabilization. Thus, it is still frequently applied to the control of electropneumatic actuators. Some of existing solutions are based on adaptive sliding mode control [12-15]. The adaptation algorithm ensures that the gain is not overestimated, which reduces the chattering. In $[16,17]$, the authors have replaced the discontinuous term by an adaptive Proportional Derivative term in order to reduce chattering phenomenon.

The main contribution of this paper is the development of a new adaptive moving sliding mode control for a class of nonlinear SISO systems which will be applied to an electropneumatic system for stroke position tracking. The proposed algorithm can guarantee a good tracking independently of the sliding surface parameters choice and enhance robustness of the control law in the presence of disturbance and parameter variations; hence the sliding surface is moved by changing the magnitude of the slopes by adaptive laws. Also, the chartering phenomenon can also be reduced by replacing the discontinuous term by an adaptive Proportional Derivative term. All parameters, adaptive laws, and sliding surface are derived based on Lyapunov stability analysis.

The remaining sections of this paper are organized as follows: in Section 2, we will present a class of nonlinear SISO systems. Section 3 is devoted to study the classic sliding mode control for the class of system under investigation. In Section 4 , we will propose an adaptive sliding mode controller 
with a moving sliding surface, and the stability analysis of the proposed method is done. Section 5 presents the description and the modeling of the pneumatic actuator system used. In Section 6, we will present the effectiveness of the proposed controller by an experimental comparative study with the classic sliding mode control. Some conclusions on the developed work and information about future work end the paper.

\section{System Formulation}

Consider a class of SISO nonlinear perturbed systems in the following form:

$$
y^{(n)}=f(\underline{x})+g(\underline{x}) U+d(t),
$$

where $\underline{x}$ is the plant state vector which is supposed to be available for measurement. $U \in \mathbb{R}$ and $y \in \mathbb{R}$ are, respectively, the system input and output. $d(t)$ is the unknown external disturbance.

Assumption 1. The upper bound of the disturbance $d(t)$ is $D$ such that $|d(t)| \leq D$.

Assumption 2. The nonlinear system (1) is assumed to be controllable $(g(\underline{x}) \neq 0)$.

Define the following tracking errors:

$$
e^{(i)}=y^{(i)}-y_{d}^{(i)}, \quad i=0, \ldots, n-1
$$

with $\left(y_{d}, \dot{y}_{d}, \ldots, y_{d}^{n-1}\right)^{T}$ being the desired reference signal vector where $(\cdot)^{(i)}$ is the successive derivatives of $(\cdot)$ given by $(\cdot)^{(i)}=d^{i}(\cdot) / d t^{i}$.

\section{Classic Sliding Mode Control}

We define a sliding surface in the space of tracking error by the following equation:

$$
S=\sum_{i=1}^{n} c_{i} e^{(i-1)} \quad \text { with } c_{n}=1
$$

The parameters $c_{n-1}, \ldots, c_{1}$ are chosen such that all roots of $h(p)=p^{(n-1)}+c_{n-1} p^{(n-2)}+\cdots+c_{2} p+c_{1}$ are in the left half of the complex plane ( $p$ denotes the complex Laplace transform variable).

Our objective is to determinate a control law $U$ for the nonlinear system given by (1) such that the vector $\left(y, \dot{y}, \ldots, y^{(n-1)}\right)^{T}$ can follow given trajectories $\left(y_{d}, \dot{y}_{d}, \ldots, y_{d}^{(n-1)}\right)^{T}$ despite the external disturbances $d(t)$.

The derivative of the sliding surface (3) is given by

$$
\dot{S}=f(\underline{x})+g(\underline{x}) U+d(t)-y_{d}^{(n)}+\sum_{i=1}^{n-1} c_{i} e^{(i)} .
$$

The SMC control law $U$ is designed as

$$
U=U_{\mathrm{eq}}+U_{\mathrm{sw}} \text {. }
$$

The equivalent law $U_{\text {eq }}$ is determined by $\dot{S}=0$. It is given by the following expression:

$$
U_{\mathrm{eq}}=g^{-1}(\underline{x})\left[-f(\underline{x})+y_{d}^{(n)}-\sum_{i=1}^{n-1} c_{i} e^{(i)}\right],
$$

where $g^{-1}(\underline{x})$ is the inverse matrix of $g(\underline{x})$. The switching term known as the discontinuous control law $U_{\text {sw }}$ is given by

$$
U_{\mathrm{sw}}=-g^{-1}(\underline{x}) U_{\mathrm{dis}}
$$

with

$$
U_{\text {dis }}=\eta \operatorname{sign}(S) \text {, }
$$

where $\eta$ is positive constant.

So the control law can be written as follows:

$$
U=g^{-1}(\underline{x})\left[-f(\underline{x})+y_{d}^{(n)}-\sum_{i=1}^{n-1} c_{i} e^{(i)}-\eta \operatorname{sign}(S)\right] \text {. }
$$

Consider the following Lyapunov function:

$$
V=\frac{1}{2} S^{2}>0
$$

The time derivative of $V$ is given by

$$
\dot{V}=S \dot{S} \text {. }
$$

By substituting (4) and (9) into (11), we obtain

$$
\dot{V}=-\eta S \operatorname{sign}(S)+S d \leq-\eta|S|+S D<-\eta|S|<0
$$

$$
\forall \eta>D \text {, }
$$

which mean that $\eta>D$ and $\dot{V}<0$; so we guarantee the semiglobal asymptotic stability and the tracking errors asymptotically converge to zero.

The sliding mode control (SMC) is characterized by its accuracy and its robustness to external disturbances. However, the principal disadvantage of this type of control is the sliding surface parameters choice and the chattering phenomenon.

In the next section, we will present an adaptive moving sliding mode controller based on time-varying linear sliding surface adaptable to arbitrary initial conditions for high order systems to have better tracking performances and an adaptive Proportional Derivative (PD) term is used to reduce the chattering phenomenon.

\section{Adaptive Moving Sliding Mode Control}

A Proportional Derivative adaptive term is substituted to the discontinuous term of SMC:

$$
U_{\mathrm{sw}}=g^{-1}(\underline{x}) U_{\mathrm{PD}} .
$$

The input and output of the continuous time PD controller are written by the following form:

$$
U_{\mathrm{PD}}=k_{p} S(t)+k_{d} \frac{d}{d t} S(t),
$$

where $k_{p}$ and $k_{d}$ are the control gains to be computed. 
The adaptive PD term derived from (14) can be rewritten as follows:

$$
U_{\mathrm{PD}}=\theta^{T} \Psi(S(t)),
$$

where $\theta$ and $\Psi(S(t))$ are, respectively, the adjustable parameters vector and the regressive vector given by $\theta=\left[\begin{array}{ll}k p & k d\end{array}\right]^{T}$ and $\Psi(S(t))=\left[\begin{array}{ll}S(t) & (d / d t) S(t)\end{array}\right]^{T}$.

The resulting control law becomes

$$
U=g^{-1}(\underline{x})\left[-f(\underline{x})+y_{d}^{(n)}-\sum_{i=1}^{n-1} \widehat{c}_{i} e^{(i)}-U_{\mathrm{PD}}\right],
$$

where $\widehat{c}_{i}$ is the estimated value of $c_{i}, i=1, \ldots, n-1$.

The parameter vectors are adjusted by the following adaptive control laws:

$$
\begin{aligned}
\dot{\vec{c}}_{i} & =\gamma_{c_{i}} S e^{(i)} \quad \text { with } i=1, \ldots, n-1, \\
\dot{\hat{\theta}} & =\gamma_{k} S \Psi(S),
\end{aligned}
$$

where $\gamma_{c_{i}}, i=1, \ldots, n-1$, and $\gamma_{k}$ are positive constants.

The main result of the proposed adaptive moving sliding mode control scheme is summarized in the following theorem.

Theorem 3. Consider the class of SISO nonlinear systems (1); the control law (16) is applied, when the parameters $\widehat{c}_{i}$ and the vector $\hat{\theta}$ are adjustable online by applying the adaptive laws (17) and (18), respectively; then the proposed control scheme guarantees the following properties:

(i) The semiglobal asymptotic stability.

(ii) Convergence to zero of the tracking errors.

Proof. We define $W_{\mathrm{PD}}$ as the approximation error:

$$
W_{\mathrm{PD}}=\eta \operatorname{sign}(S)-\theta^{T} \Psi(S(t)) .
$$

We note that $\left\|W_{\mathrm{PD}}\right\| \leq W_{\mathrm{PD}_{\max }}$. The time derivative of $S$ is given by (4). While replacing the control law $U$ by its expression (16), the time derivative of $S$ can be rewritten as

$$
\begin{aligned}
& \dot{S}=-\sum_{i=1}^{n-1} \widehat{c}_{i} e^{(i)}-U_{\mathrm{PD}}+\sum_{i=1}^{n-1} c_{i} e^{(i)}+d, \\
& \dot{S}=\sum_{i=1}^{n-1} \widetilde{c}_{i} e^{(i)}-U_{\mathrm{PD}}+d,
\end{aligned}
$$

where $\widetilde{c}_{i}=c_{i}-\widehat{c}_{i}, i=1, \ldots, n-1$.

The Lyapunov function is chosen as follows:

$$
V=\frac{1}{2} S^{2}+\frac{1}{2 \gamma_{k}} \widetilde{\theta}^{T} \widetilde{\theta}+\sum_{i=1}^{n-1} \frac{1}{2 \gamma_{c_{i}}} \widetilde{c}_{i}^{2}
$$

The time derivative of $V$ is given by

$$
\dot{V}=S \dot{S}+\frac{1}{\gamma_{k}} \widetilde{\theta}^{T} \dot{\tilde{\theta}}+\sum_{i=1}^{n-1} \frac{1}{\gamma_{c_{i}}} \widetilde{c}_{i} \dot{\tilde{c}}_{i}
$$

By replacing the derivative time of $S$ given by (20) and adding (19) in the derivative of $V$, we obtain

$$
\begin{aligned}
\dot{V} & =S\left[\sum_{i=1}^{n-1} \widetilde{c}_{i} e^{(i)}-U_{\mathrm{PD}}+d+W_{\mathrm{PD}}-\eta \operatorname{sign}(S)\right. \\
& \left.+\theta^{T} \Psi(S)\right]+\frac{1}{\gamma_{k}} \widetilde{\theta}^{T} \dot{\tilde{\theta}}+\sum_{i=1}^{n-1} \frac{1}{\gamma_{c_{i}}} \widetilde{c}_{i} \dot{\tilde{c}}_{i}=S\left[\sum_{i=1}^{n-1} \widetilde{c}_{i} e^{(i)}\right. \\
& \left.-\widehat{\theta}^{T} \Psi(S)+d+W_{\mathrm{PD}}-\eta \operatorname{sign}(S)+\theta^{T} \Psi(S)\right] \\
& +\frac{1}{\gamma_{k}} \widetilde{\theta}^{T} \dot{\tilde{\theta}}+\sum_{i=1}^{n-1} \frac{1}{\gamma_{c_{i}}} \widetilde{c}_{i} \dot{\tilde{c}}_{i}=S\left[\sum_{i=1}^{n-1} \widetilde{c}_{i} e^{(i)}+\widetilde{\theta}^{T} \Psi(S)+d\right. \\
& \left.+W_{\mathrm{PD}}-\eta \operatorname{sign}(S)\right]+\frac{1}{\gamma_{k}} \widetilde{\theta}^{T} \dot{\tilde{\theta}}^{n-1}+\sum_{i=1}^{n-1} \frac{1}{\gamma_{c_{i}}} \tilde{c}_{i} \dot{\tilde{c}}_{i} .
\end{aligned}
$$

Knowing that $\dot{\tilde{c}}_{i}=-\dot{\widehat{c}}_{i}$ and $\dot{\tilde{\theta}}=-\dot{\widehat{\theta}},(23)$ can be rewritten as follows:

$$
\begin{aligned}
\dot{V}= & S\left[\sum_{i=1}^{n-1} \widetilde{c}_{i} e^{(i)}+\tilde{\theta}^{T} \Psi(S)+d+W_{\mathrm{PD}}-\eta \operatorname{sign}(S)\right] \\
& -\frac{1}{\gamma_{k}} \widetilde{\theta}^{T} \dot{\hat{\theta}}-\sum_{i=1}^{n-1} \frac{1}{\gamma_{c_{i}}} \widetilde{c}_{i} \dot{\hat{c}}_{i} \\
= & \sum_{i=1}^{n-1} \widetilde{c}_{i}\left(S e^{(i)}-\frac{1}{\gamma_{c_{i}}} \dot{\widehat{c}}_{i}\right)+\widetilde{\theta}^{T}\left(S \Psi(S)-\frac{1}{\gamma_{k}} \dot{\hat{\theta}}\right) \\
& -\eta S \operatorname{sign}(S)+S\left(d+W_{\mathrm{PD}}\right) .
\end{aligned}
$$

By substituting (17) and (18) into (24), we obtain

$$
\begin{aligned}
& \dot{V}=-\eta S \operatorname{sign}(S)+S\left(d+W_{\mathrm{PD}}\right), \\
& \dot{V}<-\eta|S|+S\left(D+W_{\mathrm{PD}_{\max }}\right) .
\end{aligned}
$$

Finally,

$$
\dot{V}<-\eta|S|, \quad \forall \eta \geq W_{\mathrm{PD}_{\max }}+D .
$$

To prove the sliding condition, the following lemma has to be introduced.

Lemma 4 (Barbalat lemma). If $g(t): R \rightarrow R$ is uniformly continuous function for $t \geq 0$ and if the limit of the integral $\lim _{t \rightarrow \infty} \int_{0}^{t}|g(\tau)| d \tau$ exists and is finite, then $\lim _{t \rightarrow \infty} g(t)=0$.

Integrating (26) from zero to $t$, it yields

$$
V(0) \geq V(t)+\eta \int_{0}^{t}|S(\tau)| d \tau .
$$

As $t$ goes to infinity, the above integral is always less than or equal to $V(0)$. However $V(0)$ is finite and positive; then we can prove that $\lim _{t \rightarrow \infty} \int_{0}^{t}|S(\tau)| d \tau$ exists and is finite. Based on Barbalat's lemma, $S(t) \rightarrow 0$ as $t$ goes to infinity. So the sliding condition is verified. 
Remark 5. The stability result is verified if all parameters involved in (22) are bounded. To ensure the boundedness of this parameters, the adaptive laws (17) and (18) can be modified using the projection algorithm [18, 19]. The modified adaptive laws are given as follows.

For $\theta$, we use

$$
\dot{\hat{\theta}}= \begin{cases}\gamma_{k} S \Psi(S) & \text { if }\left(\|\hat{\theta}\|<M_{\theta}\right) \text { or }\left(\|\hat{\theta}\|=M_{\theta} \text { if } S \hat{\theta}^{T} \Psi(S) \geq 0\right), \\ P_{\theta}\left[\gamma_{k} S \Psi(S)\right] & \text { if }\left(\|\hat{\theta}\|=M_{\theta}\right),\left(S \hat{\theta}^{T} \Psi(S)>0\right) .\end{cases}
$$

For $c_{i}$, we use

$$
\dot{\hat{c}}_{i}= \begin{cases}\gamma_{c_{i}} S e^{(i)} & \text { if }\left(\left\|\widehat{c}_{i}\right\|<M_{\widehat{c}_{i}}\right) \text { or }\left(\left\|\widehat{c}_{i}\right\|=M_{\widehat{c}_{i}} \text { if } S \widehat{c}_{i} e^{(i)} \geq 0\right), \\ P_{c_{i}}\left[\gamma_{c_{i}} S e^{(i)}\right] & \text { if }\left(\left\|\widehat{c}_{i}\right\|=M_{\widehat{c}_{i}}\right),\left(S \widehat{c}_{i} e^{(i)}>0\right),\end{cases}
$$

where $M_{\theta}$ and $M_{\widehat{c}_{i}}$ are the design parameters that specify the allowable bounds of $\widehat{\theta}$ and $\widehat{c}_{i}$.

The projection operators $P_{\theta}[*]$ and $P_{c_{i}}[*]$ are defined as

$$
\begin{gathered}
P_{\theta}\left[\gamma_{k} S \Psi(S)\right]=\gamma_{k} S \Psi(S)-\gamma_{k} S \frac{\widehat{\theta} \widehat{\theta}^{T} \Psi(S)}{\|\hat{\theta}\|^{2}}, \\
P_{c_{i}}\left[\gamma_{c_{i}} S e^{(i)}\right]=\gamma_{c_{i}} S e^{(i)}-\gamma_{c_{i}} S \frac{\widehat{c}_{i} \widehat{c}_{i}^{T} e^{(i)}}{\left\|\widehat{c}_{i}\right\|^{2}}
\end{gathered}
$$

\section{Application to Pneumatic Actuator Position Control}

5.1. Pneumatic Actuator Modeling. The electropneumatic system (Figure 1) is a linear double acting actuator. It is composed of two chambers denoted by $P$ and $N$. Two threeway servodistributors supply the air mass flow rates entering into the two chambers. The cylinder axis is connected at its extremity to a carriage and carries a load on guiding rails. The full moving mass is $17 \mathrm{Kg}$.

According to Newton's second law applied to the moving solid and under the assumptions of a link rod/rigid carriage and a rigid body, the dynamic equation of movement is

$$
\begin{aligned}
& \frac{d y}{d t}=v, \\
& \frac{d v}{d t}=\frac{1}{M}\left[S_{P} p_{P}-S_{N} p_{N}-F_{\mathrm{vis}}(v)-F_{\mathrm{ext}}\right],
\end{aligned}
$$

where $y$ and $v$ are, respectively, the position and the velocity of the rod and $p_{P}$ and $p_{N}$ stand, respectively, for the pressure in chambers $P$ and $N$.

$F_{\text {vis }}$ are viscous friction forces which are proportional to the velocity by a coefficient of viscosity $b_{v}$ :

$$
F_{\text {vis }}=b_{v} v
$$

The external forces $F_{\text {ext }}$ are considered constant, and they represent the action of the atmospheric pressure $P_{E}$ on the moving part:

$$
F_{\text {ext }}=\left(S_{P}-S_{N}\right) P_{E}
$$

To obtain the pressure evolution in a chamber with variable volume, we assume the following assumptions [20]:

(i) The air is supposed to be a perfect gas.

(ii) The pressure and the temperature are homogeneous in each chamber.

(iii) The process is polytropic and characterized by a coefficient $k$.

(iv) The temperature variation is very small with respect to average and equal to the supply temperature $\left(T_{p}=\right.$ $\left.T_{N}=T_{s}\right)$.

The servodistributors dynamic can be neglected. So we can reduce the servodistributors model to a static one which can be described by two relationships $q_{m P}\left(u, p_{P}\right)$ and $q_{m N}\left(-u, p_{N}\right)$ between the mass flow rates $q_{m P}$ and $q_{m N}$; the input voltages $u$; and the output pressures $p_{P}$ and $p_{N}$. The pressure dynamics in both chambers $P$ and $N$ are given as follows:

$$
\begin{aligned}
& \frac{d p_{P}}{d t}=\frac{k r T_{s}}{V_{P}(y)}\left[q_{m P}\left(u, p_{P}\right)-\frac{S_{P}}{r T_{s}} p_{P} v\right], \\
& \frac{d p_{N}}{d t}=\frac{k r T_{s}}{V_{N}(y)}\left[q_{m N}\left(-u, p_{N}\right)+\frac{S_{N}}{r T_{s}} p_{N} v\right] .
\end{aligned}
$$

$V_{P}$ and $V_{N}$ are, respectively, the volumes of $P$ and $N$ chambers:

$$
\begin{aligned}
& V_{P}(y)=V_{P}(0)+S_{P} y, \\
& V_{N}(y)=V_{N}(0)-S_{N} y
\end{aligned}
$$




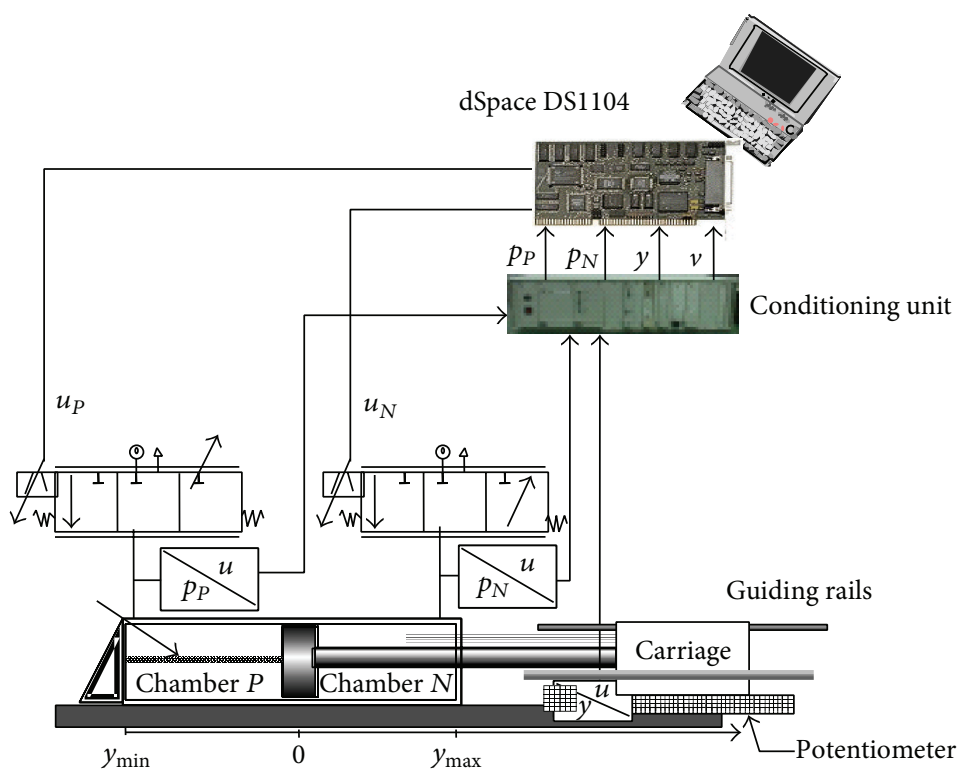

FIGURE 1: Electropneumatic system.

with

$$
\begin{gathered}
V_{P}(0)=V_{D P}+S_{P} \frac{l}{2}, \\
V_{N}(0)=V_{D N}+S_{N} \frac{l}{2},
\end{gathered}
$$

where $V_{D[P \text { or } N]}$ are dead volumes present in each extremity of the cylinder.

5.2. Control Model. In [21], authors have proposed analytical models for both simulation and control purposes. The flow stage characteristics were approximated by affine polynomial functions in control such that

$$
q_{m}(u, p)=\varphi(p)+\psi(p, \operatorname{sign}(u)) u .
$$

The polynomial functions $\varphi(p), \psi(p, u>0)$, and $\psi(p, u<0)$ degrees are equal to five. The maximum error of mass flow rate between polynomial approximation and static measurement is less than $10 \%$.

The nonlinear affine model (38) is obtained referring to (31), (34), and (37). Consider the following:

$$
\begin{aligned}
\frac{d y}{d t}=v \\
\frac{d v}{d t}=\frac{1}{M}\left[S_{P} p_{P}-S_{N} p_{N}-F_{\mathrm{vis}}(v)-F_{\mathrm{ext}}\right], \\
\frac{d p_{P}}{d t}=\frac{k r T_{s}}{V_{P}(y)}\left[\varphi\left(p_{P}\right)-\frac{S_{P}}{r T_{s}} p_{P} v\right] \\
+\frac{k r T_{s}}{V_{P}(y)} \psi\left(p_{P}, \operatorname{sgn}(u)\right) u,
\end{aligned}
$$

$$
\begin{aligned}
\frac{d p_{N}}{d t}= & \frac{k r T_{s}}{V_{N}(y)}\left[\varphi\left(p_{N}\right)+\frac{S_{N}}{r T_{s}} p_{N} v\right] \\
& -\frac{k r T_{s}}{V_{N}(y)} \psi\left(p_{N}, \operatorname{sgn}(-u)\right) u,
\end{aligned}
$$

where $k$ is the polytropic coefficient.

From (38), it is clear that the actuator position $y$ has a relative degree equal to three versus the control input $u$. The time derivative of the acceleration $a$ is given as follows:

$$
\begin{aligned}
\dot{a}= & \frac{k r T_{s}}{M}\left(\frac{S_{P}}{V_{P}} \varphi_{P}-\frac{S_{N}}{V_{N}} \varphi_{N}\right) \\
& -\left(\frac{k S_{P}^{2}}{M V_{P}} p_{P} v+\frac{k S_{N}^{2}}{M V_{N}} p_{N} v\right)-\frac{b_{v}}{M} a \\
& +\left[\frac{S_{P} k}{M V_{P}}\left(r T_{s} \psi_{P}\right)+\frac{S_{N} k}{M V_{N}}\left(r T_{s} \psi_{N}\right)\right] u .
\end{aligned}
$$

Equation (39) can be written under the class of SISO nonlinear system described by (1):

$$
y^{(3)}=f(\underline{x})+g(\underline{x}) U,
$$

where $\underline{x}=\left[\begin{array}{lllll}y & v & a & p_{P} & p_{N}\end{array}\right]^{T}$ is the state vector composite of position, velocity, and acceleration of the pneumatic actuator, as well as pressures inside both cylinder's chambers:

$$
\begin{aligned}
f(\underline{x})= & \frac{k r T_{s}}{M}\left(\frac{S_{P}}{V_{P}} \varphi_{P}-\frac{S_{N}}{V_{N}} \varphi_{N}\right) \\
& -\left(\frac{k S_{P}^{2}}{M V_{P}} p_{P} v+\frac{k S_{N}^{2}}{M V_{N}} p_{N} v\right)-\frac{b_{v}}{M} a, \\
g(\underline{x})= & \frac{k r T_{s}}{M}\left(\frac{S_{P}}{V_{P}} \psi_{P}+\frac{S_{N}}{V_{N}} \psi_{N}\right) .
\end{aligned}
$$


To proceed to the control of system, we choose $y$ as the output of the system which is the position of the pneumatic actuator.

5.3. Classic Sliding Mode Control. The sliding surface defined by (3) is given by the following expression:

$$
S=c_{1} e+c_{2} \dot{e}+\ddot{e}
$$

with

$$
\begin{aligned}
& e=y-y_{d}, \\
& \dot{e}=\dot{y}-\dot{y}_{d}=v-v_{d}, \\
& \ddot{e}=\ddot{y}-\ddot{y}_{d}=a-a_{d},
\end{aligned}
$$

where $y^{d}, v^{d}$, and $a^{d}$ are, respectively, the position, velocity, and acceleration desired trajectories. obtain

By applying the control law given by expression (9), we

$$
U=g^{-1}(\underline{x})\left[-f(\underline{x})+y_{d}^{(3)}-c_{1} \dot{e}-c_{2} \ddot{e}-\eta \operatorname{sign}(S)\right] .
$$

5.4. Adaptive Moving Sliding Mode Control. Now, we apply the proposed AMSMC to the pneumatic actuator. The control law is given by the following expression:

\section{$U$}

$$
=g^{-1}(\underline{x})\left[-f(\underline{x})+y_{d}^{(3)}-\widehat{c}_{1} \dot{e}-\widehat{c}_{2} \ddot{e}-\widehat{\theta}^{T} \Psi(S(t))\right] .
$$

The adaptive parameters of the control law (45) are given by

$$
\begin{aligned}
& \dot{\hat{c}}_{1}=\gamma_{c_{1}} S \dot{e}, \\
& \dot{\hat{c}}_{2}=\gamma_{c_{2}} S \ddot{e}, \\
& \dot{\hat{\theta}}=\gamma_{k} S \Psi(S),
\end{aligned}
$$

where $\gamma_{c_{1}}, \gamma_{c_{2}}$, and $\gamma_{k}$ are positives constants.

\section{Experimental Results}

The adaptive moving sliding mode controller applied to an electropneumatic system was implemented using dSpace DS1104 controller board with a dedicated digital signal processor. The measured signals are all analogical. They are transmitted via a signal conditioning unit before being read by 16-bit Analog Digital Converter (ADC). We choose the sampling time equal to $1 \mathrm{~ms}$. Experiment results are carried out here to demonstrate the effectiveness of the adaptive controller.

In order to obtain velocity feedback, there exist two ways: using a velocity sensor or recovering velocity signal using position information. To reduce the cost of the system, the velocity of the cylinder is determined by analogically differentiating and low-pass filtering the position. All control laws are implemented by using a position sensor and two pressure sensors.

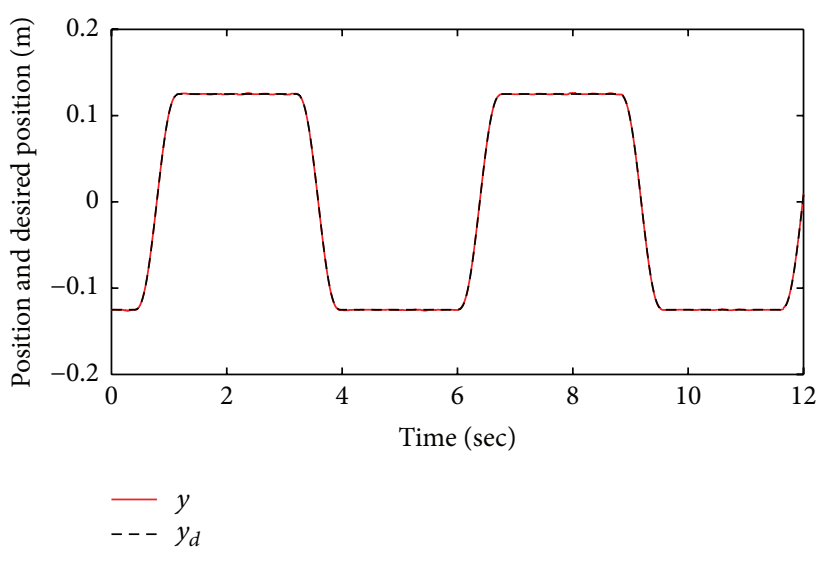

FIgure 2: Position and desired position (m).

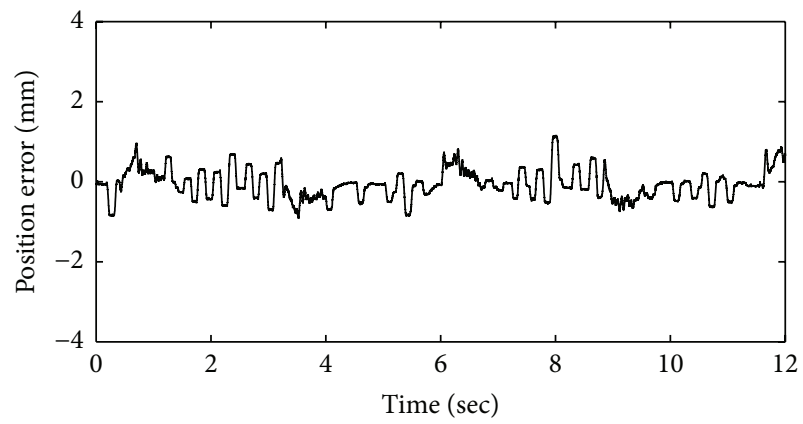

Figure 3: Position error ( $\mathrm{mm})$.

The position sensor is a NovoTECHNIK, model TLH500. Its linearity error is $\pm 0.05 \%$. The two pressure sensors are fabricated by KULITE model XT140M-7BarA, their precision is equal to $700 \mathrm{~Pa}(0.1 \%)$, and their combined nonlinearity and hysteresis errors are $\pm 0.1 \%$.

The experiment results related to the pneumatic system controllers are given by Figures 2-13. In order to show the proposed controller efficiency, we compare the experiment results of sliding mode control (SMC) (Figures 2-4) with those of adaptive moving sliding mode control (AMSMC) (Figures 7-13).

6.1. Experiment Results of Classic Sliding Mode Control. First, the control gain is chosen as $\eta=50$. Figures 2 and 3 show that the SMC has good tracking position performance. The maximum position error is about $1.2 \mathrm{~mm}(0.48 \%$ of the total displacement magnitude). In static stage, the position error is about $400 \mu \mathrm{m}$.

In [2], a hybrid of fuzzy and PID control algorithm is proposed for point-to-point displacement. In this case, the steady-state error is about $3.5 \mathrm{~mm}$ (the total displacement is equal to $200 \mathrm{~mm}$ ). A PID controller augmented with friction compensation using neural network is presented in [22]. A sinusoid with magnitude of $70 \mathrm{~mm}$ and frequency of $0.2 \mathrm{~Hz}$ has been used as reference input. In this case, the maximum position error is about $8.1 \mathrm{~mm}$. So, from this point of view, the obtained results with the SMC controller are more attractive. 


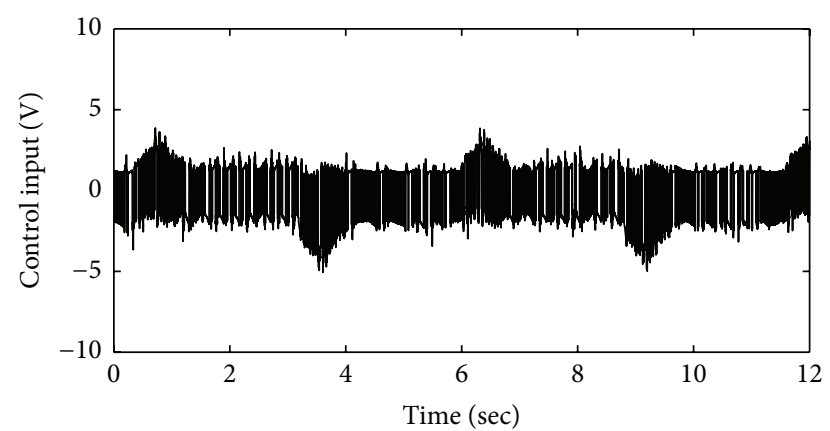

Figure 4: Control input $u(\mathrm{~V})$.

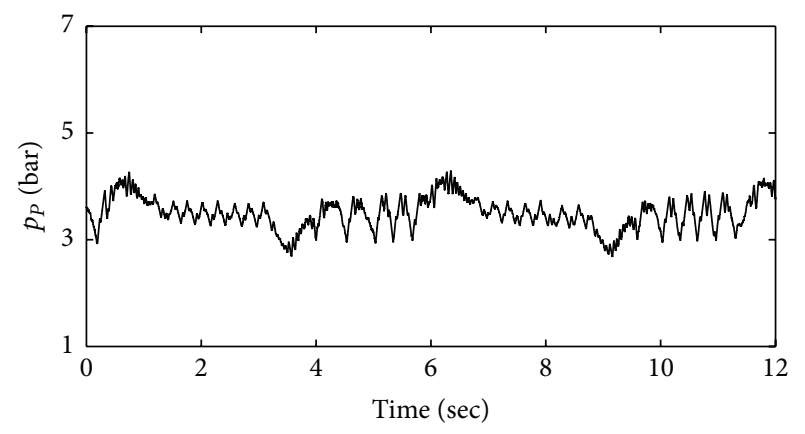

Figure 5: Pressure $p_{P}$ (bar).

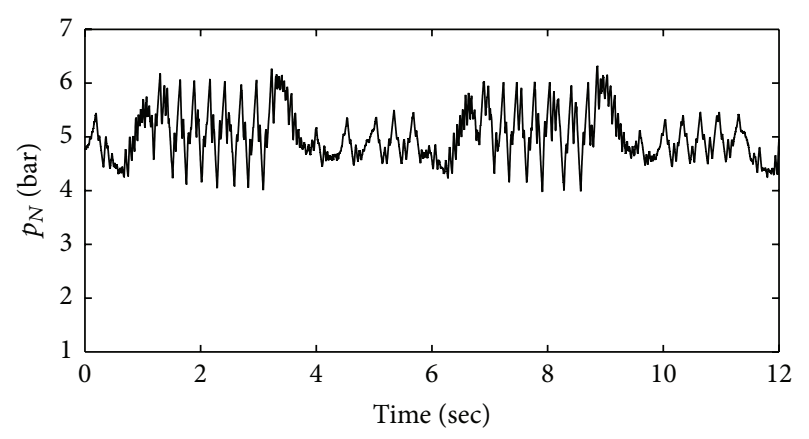

Figure 6: Pressure $p_{N}$ (bar).

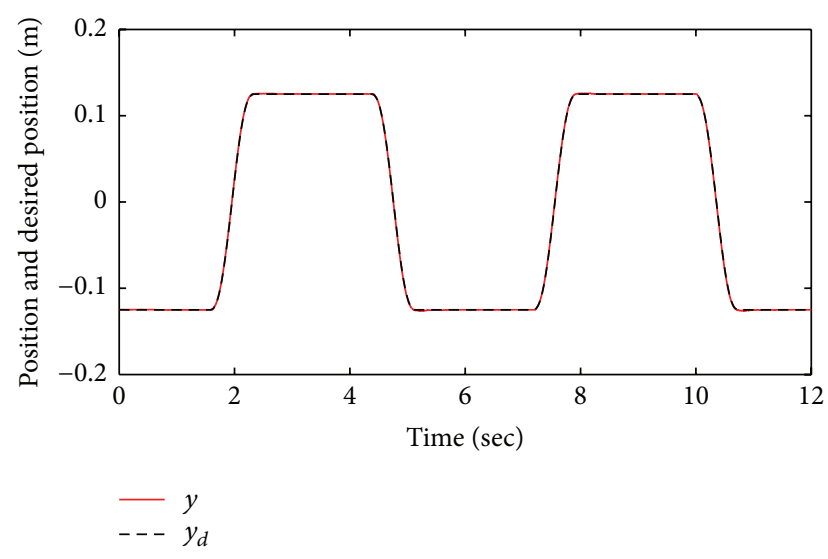

Figure 7: Position and desired position (m).

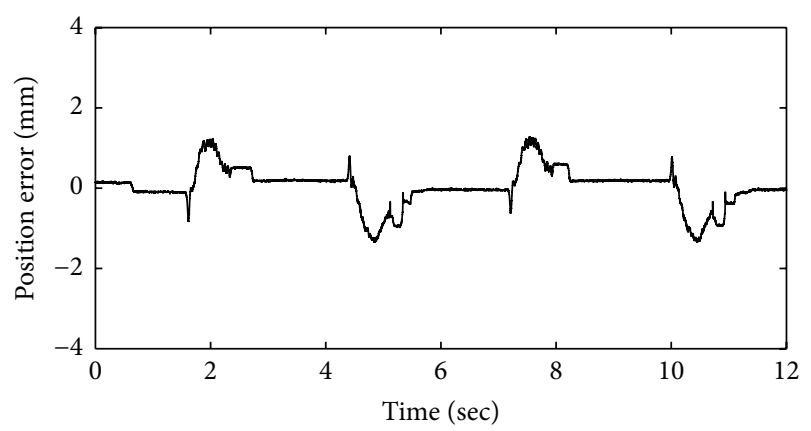

FIgURE 8: Position error (mm).

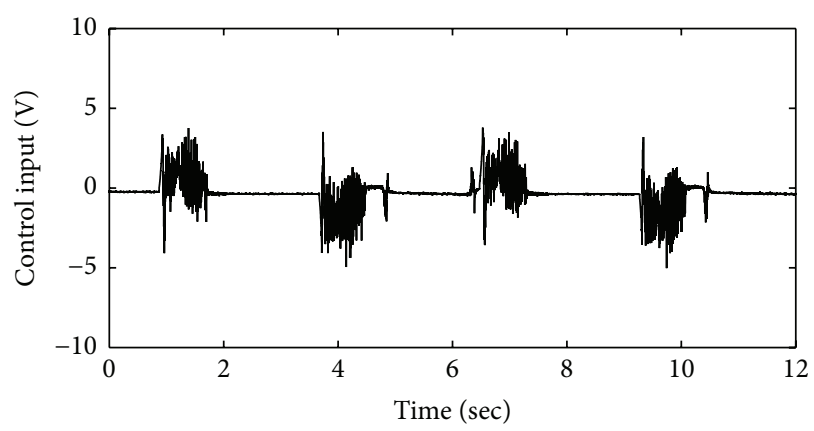

Figure 9: Control input $u(\mathrm{~V})$.

However the major drawback presented by the SMC is the chattering phenomenon (see Figure 4). This phenomenon is undesirable and it can be responsible for the life time drop of some complements like the servodistributor.

Figures 5 and 6 show that the pressure evolutions in chambers $P$ and $N$ are stiff and present a high-frequency excitation.

6.2. Experiment Results of Adaptive Moving Sliding Mode Control. As shown in Figures 7 and 8, the AMSMC have better tracking position performance than the SMC. In steady state, the position error is about $160 \mu \mathrm{m}$ and the maximum position error is about $1.3 \mathrm{~mm}(0.52 \%$ of the total displacement magnitude). Moreover, the chattering phenomenon in the control law (see Figure 9) has been reduced. The pressure evolutions are smooth and without high-frequency excitation (see Figures 10 and 11). Figures 12 and 13 illustrate the behavior adaptation parameters and sliding surface gains. Further, we note that the sliding surface Figure 14 converges to zero in finite time. So, from this point of view, the AMSMC controller seems to be more useful than the SMC.

Table 1 represents a comparative study of different control strategy applied such as the backstepping controller [4], the passivity control [23], the high order SMC [24], and the two control laws presented in this paper (SMC and AMSMC). Those control laws have been implemented on the same benchmark, on the same condition, and on the same setup condition. The signs + and - in Table 1 mean that the criterion is satisfied and dissatisfied, respectively, and the sign ++ means that the criterion is very satisfied. 
TABLE 1: Comparative study of different control strategy.

\begin{tabular}{lccc}
\hline $\begin{array}{l}\text { Control } \\
\text { strategy }\end{array}$ & $\begin{array}{c}\text { Maximum } \\
\text { position error in } \\
\text { dynamic stage } \\
(\mathrm{mm})\end{array}$ & $\begin{array}{c}\text { Mean position } \\
\text { error in static } \\
\text { stage }(\mathrm{mm})\end{array}$ & Chattering \\
\hline $\begin{array}{l}\text { Backstepping } \\
\text { Passivity }\end{array}$ & 1.62 & 0.13 & ++ \\
$\begin{array}{l}\text { control } \\
\text { SMC }\end{array}$ & 1.27 & 0.11 & + \\
$\begin{array}{l}\text { High order } \\
\text { SMC }\end{array}$ & 1.2 & 0.4 & - \\
AMSMC & 1.9 & 0.11 & + \\
\hline
\end{tabular}

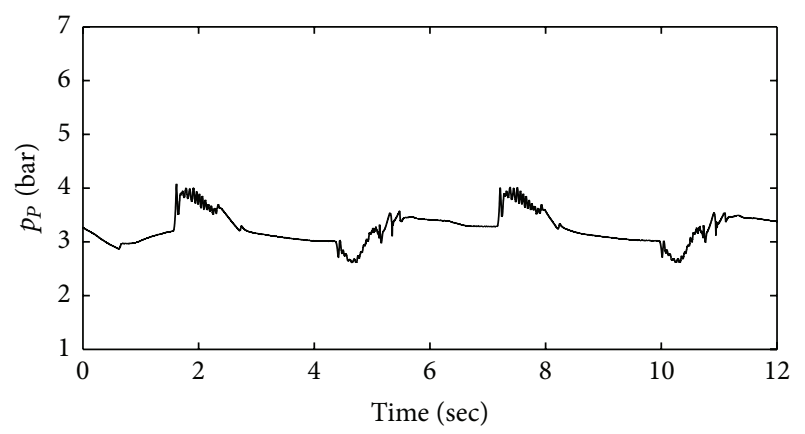

Figure 10: Pressure $p_{P}$ (bar).

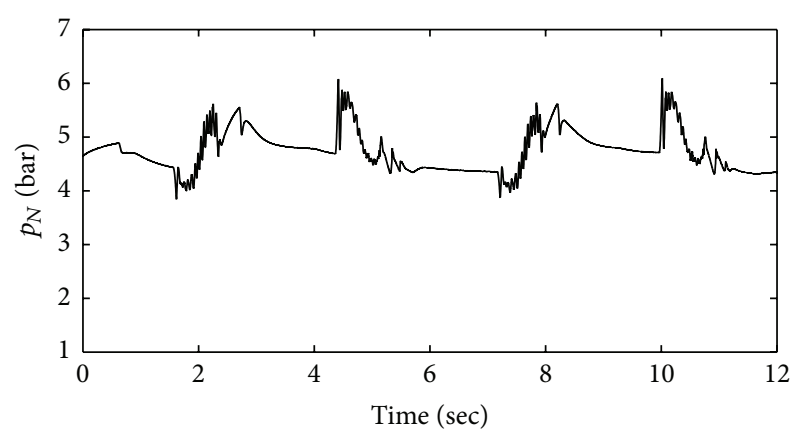

Figure 11: Pressure $p_{N}$ (bar).

Based on Table 1, the sliding mode control is an attractive and robust approach; it has the less maximum position error in dynamic stage $1.2 \mathrm{~mm}$ but it suffers from specific drawback which is the chattering phenomenon. This phenomenon is undesirable and seems to be responsible for the lifetime drop of some components especially the servodistributor. There are many control strategies to reduce the chattering phenomenon. From Table 1, it is clear that backstepping controller and AMSMC present the important solution to eliminate this phenomenon. Using the backstepping controller, the maximum position error in dynamic stage is about $1.62 \mathrm{~mm}$ and for the proposed controller is about $1.3 \mathrm{~mm}$. From experimental results and Table 1, we can conclude that the AMSMC ensures a good accuracy in terms of position tracking for a desired trajectory and remove the chattering effect.
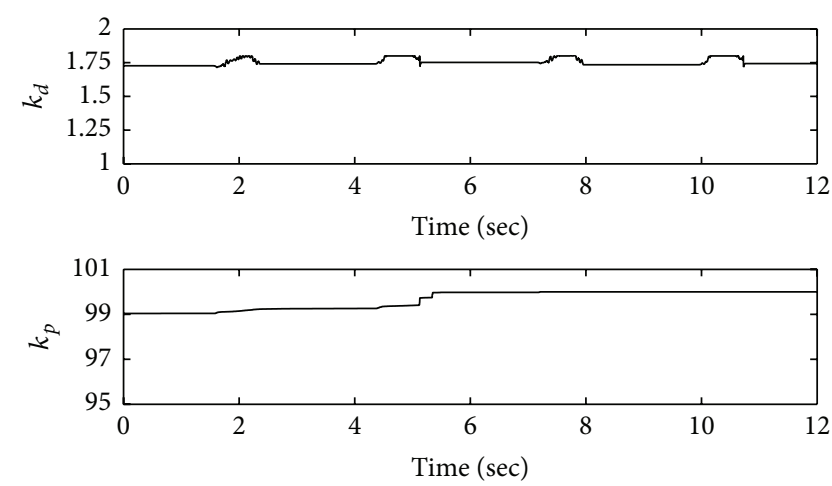

Figure 12: Adaptation parameters $k_{p}$ and $k_{d}$.
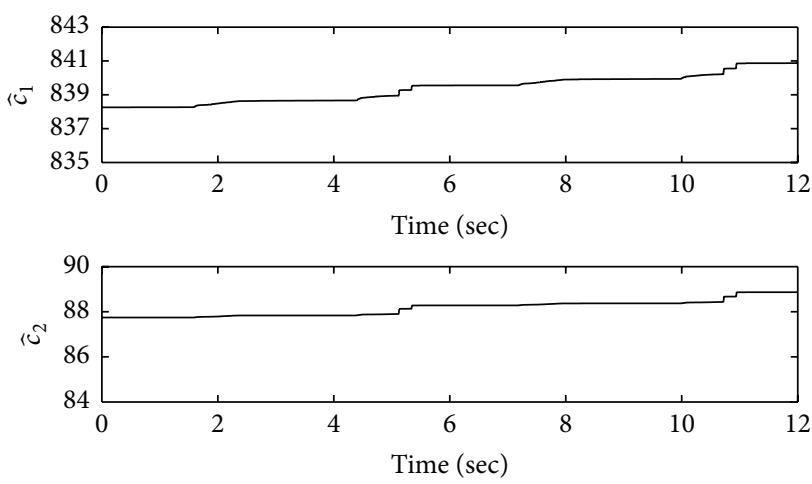

FIGURE 13: Surface parameters $c_{1}$ and $c_{2}$.

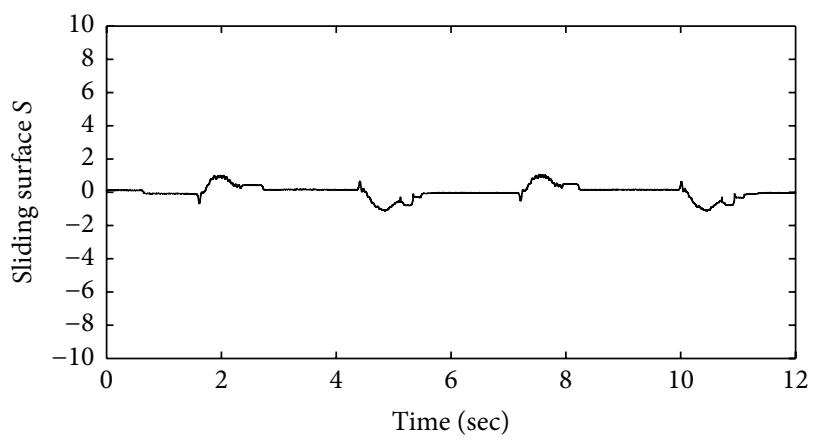

FIgURE 14: Sliding surface $S$.

\section{Conclusion}

In this paper, an adaptive moving sliding mode controller is designed for a class of nonlinear single-input single-output systems. In order to ensure a good accuracy of tracking desired reference signal and to overcome the chattering problem, on the one hand, we proposed a moving sliding surface which is moved by changing the magnitude of the slope and intercept towards a predetermined surface, and, on the other hand, we combined an adaptive PD term into classic sliding mode controller. The proposed control scheme has guaranteed the global stability based on Lyapunov stability approach. To show the performance and the effectiveness of our proposed controller, experimental results related to the 
electropneumatic system control were presented and compared with the classic sliding mode controller. The AMSMC controller which reduced the chattering phenomenon still provides a good tracking performance. Future works will extend our controller for a class of nonlinear Multi-Input Multi-Output (MIMO) systems.

\section{Nomenclatures}

$\begin{array}{ll}y, v, a: \begin{array}{l}\text { Position }(\mathrm{m}), \text { velocity }(\mathrm{m} / \mathrm{s}), \text { and } \\ \text { acceleration }\left(\mathrm{m} / \mathrm{s}^{2}\right)\end{array} \\ j: & \text { Jerk }\left(\mathrm{m} / \mathrm{s}^{3}\right) \\ p_{P, N}: & \text { Pressure in chambers } P \text { and } N(\mathrm{~Pa}) \\ p_{E}: & \text { Exhaust pressure }(\mathrm{Pa}) \\ u_{P}, u_{N}: & \text { Servodistributors voltages }(\mathrm{V}) \\ q_{m}: & \text { Mass flow rate }(\mathrm{kg} / \mathrm{s}) \\ \varphi(\cdot): & \text { Leakage polynomial function }(\mathrm{kg} / \mathrm{s}) \\ \psi(\cdot): & \text { Polynomial function }(\mathrm{kg} / \mathrm{s} / \mathrm{V}) \\ b: & \text { Viscous friction coefficient }(\mathrm{N} / \mathrm{m} / \mathrm{s}) \\ F_{\text {ext }}: & \text { External force }(\mathrm{N}) \\ k: & \text { Polytropic constant } \\ l: & \text { Length of stroke }(\mathrm{m}) \\ M: & \text { Total load mass }(\mathrm{kg}) \\ S: & \text { Piston } P, N \text { section } \\ T: & \text { Temperature K } \\ r: & \text { Perfect gas constant }(\mathrm{J} / \mathrm{kg} / \mathrm{K}) .\end{array}$

\section{Competing Interests}

The authors declare that there is no conflict of interests regarding the publication of this paper.

\section{Acknowledgments}

This work was supported by the Ministry of Higher Education and Scientific Research of Tunisia.

\section{References}

[1] T. Kimura, S. Hara, T. Fujita, and T. Kagawa, "Feedback linearization for pneumatic actuator systems with static friction," Control Engineering Practice, vol. 5, no. 10, pp. 1385-1394, 1997.

[2] M. Parnichkun and C. Ngaecharoenkul, "Kinematics control of a pneumatic system by hybrid fuzzy PID," Mechatronics, vol. 11, no. 8, pp. 1001-1023, 2001 .

[3] B. Li, Z. Li, and Y. Xu, "Study on adaptive control for a pneumatic position servo system," Advances in Modelling and Analysis, vol. 49, no. 2, pp. 21-28, 1997.

[4] M. Smaoui, X. Brun, and D. Thomasset, "A study on tracking position control of an electropneumatic system using backstepping design," Control Engineering Practice, vol. 14, no. 8, pp. 923933, 2006.

[5] M. Zribi, H. Sira-Ramirez, and A. Ngai, "Static and dynamic sliding mode control schemes for a permanent magnet stepper motor," International Journal of Control, vol. 74, no. 2, pp. 103117, 2001.

[6] M. Smaoui, X. Brun, and D. Thomasset, "Systematic control of an electropneumatic system: integrator backstepping and sliding mode control," IEEE Transactions on Control Systems Technology, vol. 14, no. 5, pp. 905-913, 2006.

[7] A. Girin, F. Plestan, X. Brun, and A. Glumineau, "A 3rd order sliding mode controller based on integral sliding mode for an electropneumatic system," in Proceedings of the 45th IEEE Conference on Decision and Control (CDC '06), pp. 1617-1622, San Diego, Calif, USA, December 2006.

[8] S. Laghrouche, M. Smaoui, F. Plestan, and X. Brun, "Higher order sliding mode control based on optimal approach of an electropneumatic actuator," International Journal of Control, vol. 79, no. 2, pp. 119-131, 2006.

[9] A. Girin, F. Plestan, X. Brun, and A. Glumineau, "Highorder sliding-mode controllers of an electropneumatic actuator: application to an aeronautic benchmark," IEEE Transactions on Control Systems Technology, vol. 17, no. 3, pp. 633-645, 2009.

[10] F. Plestan, E. Moulay, and A. Glumineau, "Output feedback sampling control: a robust solution based on second order sliding mode," in Proceedings of the 48th IEEE Conference on Decision and Control Held Jointly with the 28th Chinese Control Conference (CDC/CCC '09), pp. 8423-8427, Shanghai, China, December 2009.

[11] J. Ding and H. Yang, "Adaptive iterative learning control for a class of uncertain nonlinear systems with second-order sliding mode technique," Circuits, Systems, and Signal Processing, vol. 33, no. 6, pp. 1783-1797, 2014.

[12] Y. Shtessel, M. Taleb, and F. Plestan, "A novel adaptive-gain supertwisting sliding mode controller: methodology and application," Automatica, vol. 48, no. 5, pp. 759-769, 2012.

[13] Z. Wang, L. Huang, X. Yang, and A. Xin, "Adaptive fuzzy control for stochastic nonlinear systems via sliding mode method," Circuits, Systems, and Signal Processing, vol. 32, no. 6, pp. 28392850, 2013.

[14] F. Plestan, Y. Shtessel, V. Brégeault, and A. Poznyak, "Sliding mode control with gain adaptation: application to an electropneumatic actuator," Control Engineering Practice, vol. 21, no. 5, pp. 679-688, 2013.

[15] S. Mondal and C. Mahanta, "Adaptive second order terminal sliding mode controller for robotic manipulators," Journal of the Franklin Institute, vol. 351, no. 4, pp. 2356-2377, 2014.

[16] S. Aloui, O. Pagès, A. El Hajjaji, A. Chaari, and Y. Koubaa, "Improved fuzzy sliding mode control for a class of MIMO nonlinear uncertain and perturbed systems," Applied Soft Computing, vol. 11, no. 1, pp. 820-826, 2011.

[17] S. Larguech, S. Aloui, O. Pages, A. E. Hajjaji, and A. Chaari, "Adaptive sliding mode control for a class of nonlinear MIMO systems: application to a turbocharged diesel engine," in Proceedings of the 22nd Mediterranean Conference of Control and Automation (MED '14), pp. 1205-1210, IEEE, Palermo, Italy, June 2014.

[18] H. F. Ho, Y. K. Wong, and A. B. Rad, "Adaptive fuzzy sliding mode control with chattering elimination for nonlinear SISO systems," Simulation Modelling Practice and Theory, vol. 17, no. 7, pp. 1199-1210, 2009.

[19] I. F. Jasim, P. W. Plapper, and H. Voos, "Adaptive sliding mode fuzzy control for unknown robots with arbitrarily-switched constraints," Mechatronics, vol. 30, pp. 174-186, 2015.

[20] J. L. Sheare, "Study of pneumatic processes in the continuous control of motion with compressed air," Transactions of the American Society of Mechanical Engineers, vol. 78, pp. 233-249, 1956.

[21] M. Belgharbi, D. Thomasset, S. Scavarda, and S. Sesmat, "Analytical model of the flow stage of a pneumatic servodistributor 
for simulation and nonlinear control," in Proceedings of the 6th Scandinavian International Conference on Fluid Power, pp. 847860, Tampere, Finland, 1996.

[22] H. K. Lee, G. S. Choi, and G. H. Choi, "A study on tracking position control of pneumatic actuators," Mechatronics, vol. 12, no. 6, pp. 813-831, 2002.

[23] K. Turki, M. Smaoui, D. Thomasset, F. Mnif, and N. Derbel, "Nonlinear passivity based control law with application to electropneumatic system," in Proceedings of the 17th IFAC World Congress, pp. 1827-1832, Seoul, Republic of Korea, July 2008.

[24] M. Smaoui, X. Brun, and D. Thomasset, "High-order sliding mode for an electropneumatic system: a robust differentiatorcontroller design," International Journal of Robust and Nonlinear Control, vol. 18, no. 4-5, pp. 481-501, 2008. 


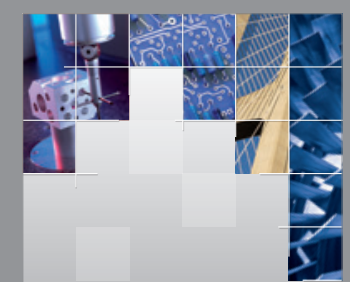

\section{Enfincering}
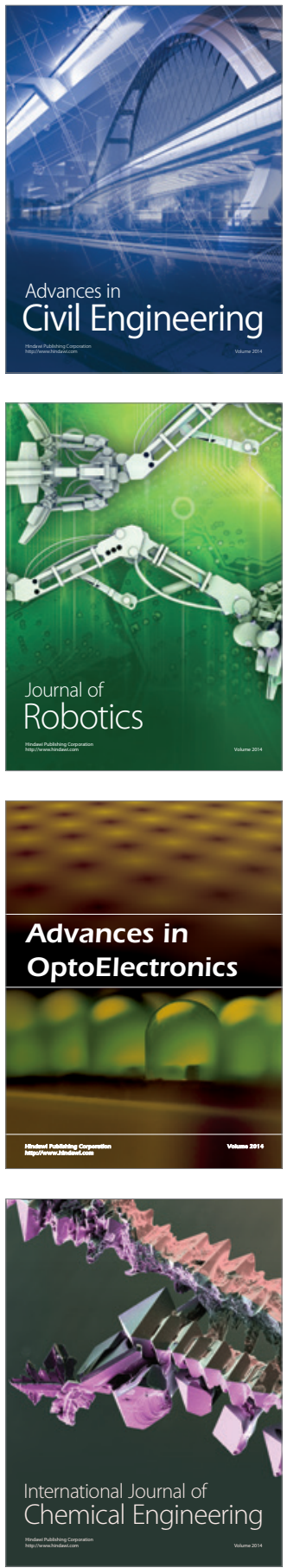

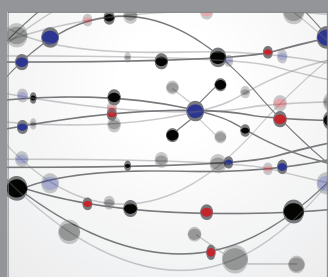

The Scientific World Journal

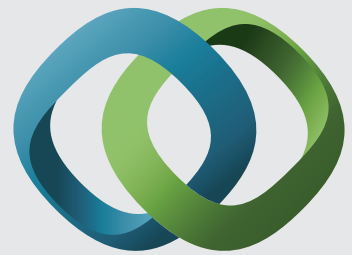

\section{Hindawi}

Submit your manuscripts at

http://www.hindawi.com
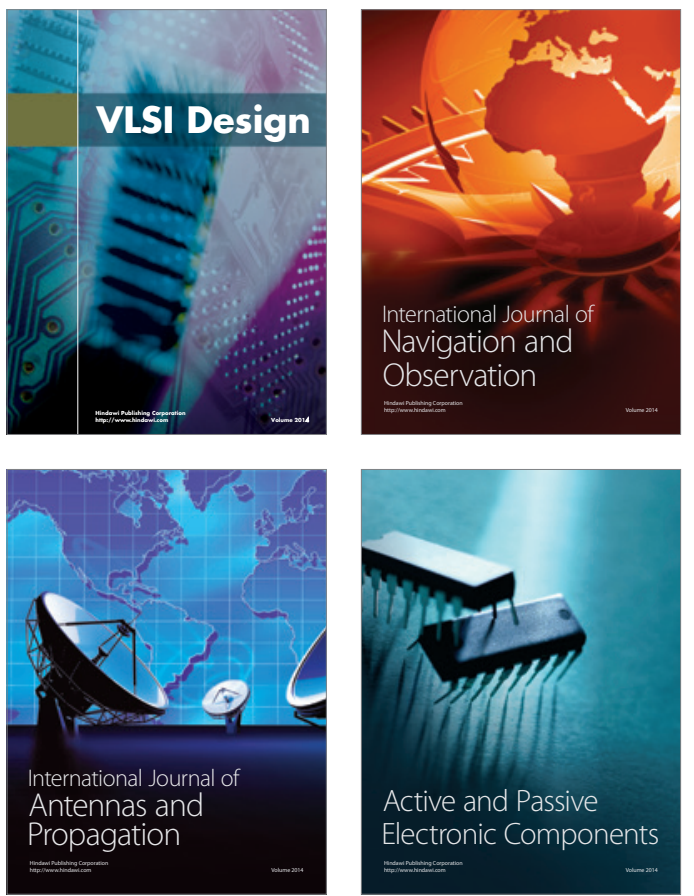
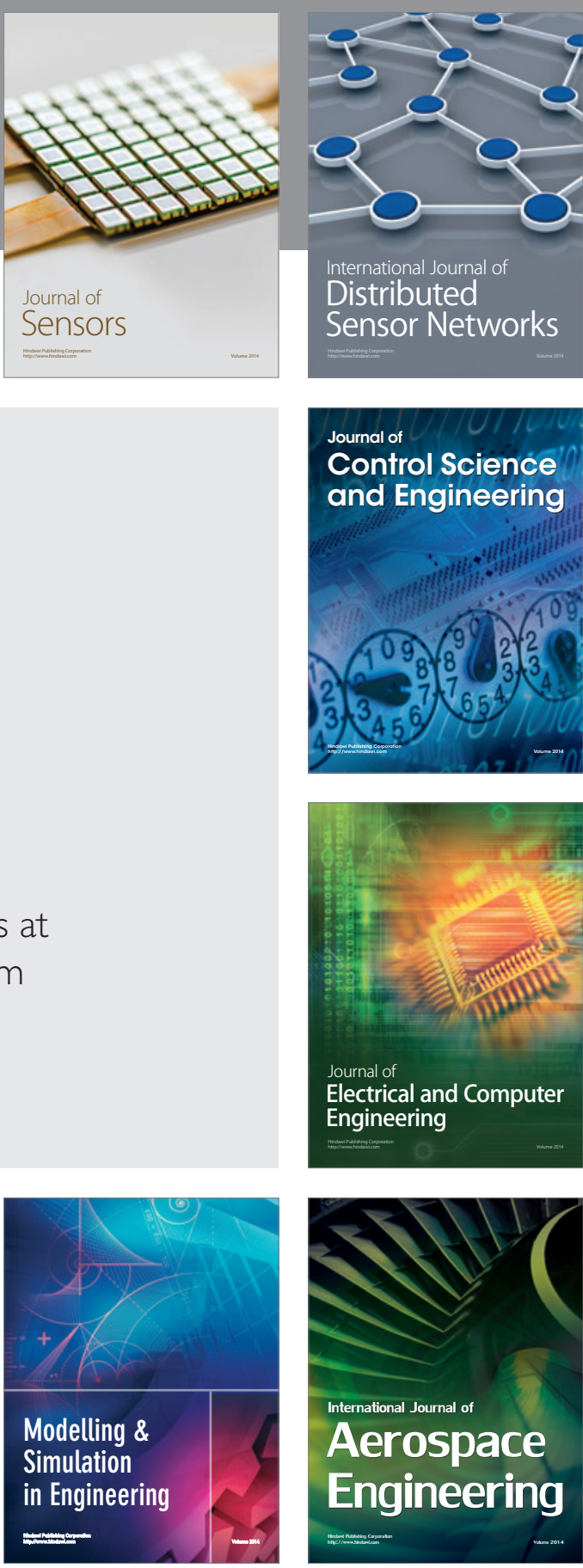

International Journal of

Distributed

Sensor Networks

Journal of

Control Science

and Engineering
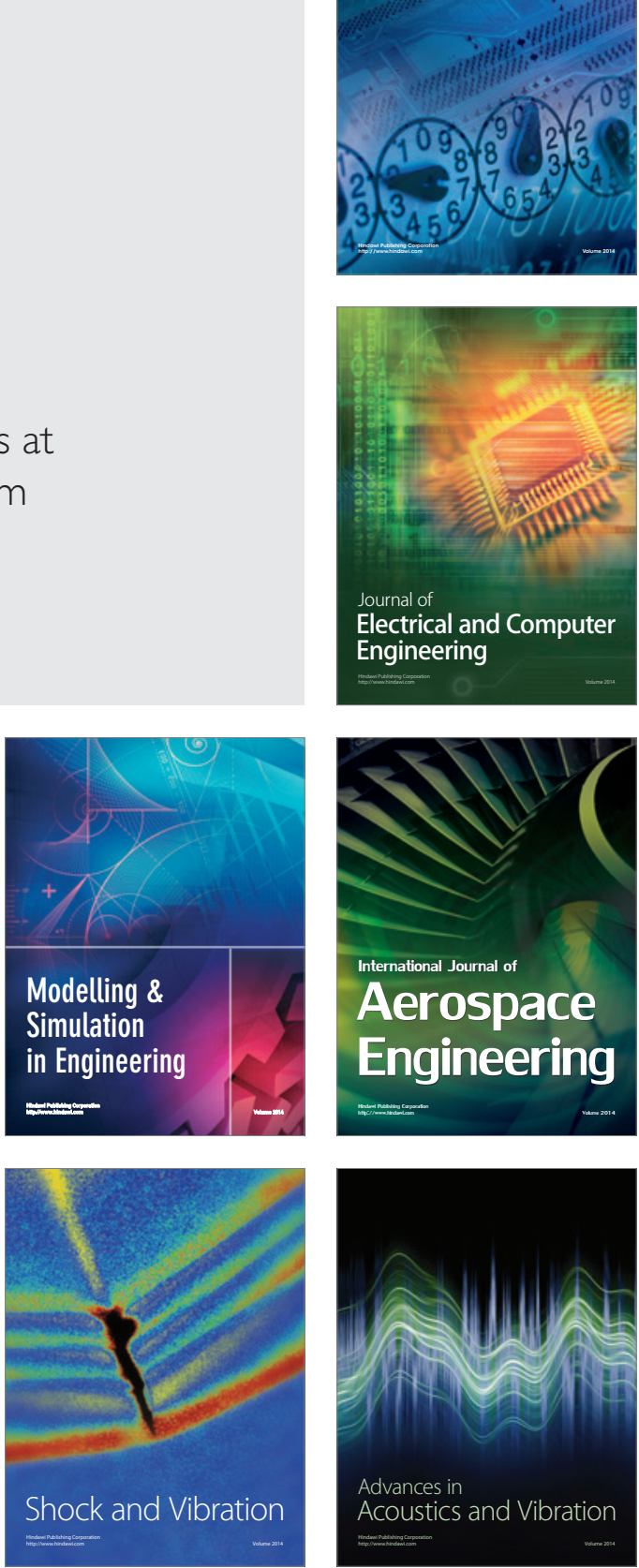